\title{
Risk Assessment of Wind Generation Dispatch Using Monte Carlo Simulation
}

\author{
Mohamed Shaaban *, M. D. Usman \\ Centre of Electrical Energy Systems (CEES), Faculty of Electrical Engineering, Universiti Teknologi Malaysia, \\ 81310 UTM, Johor Bahru, Malaysia
}

\begin{abstract}
The risk associated with introducing wind turbines into the generation fleet is investigated in this paper. The loss of load probability (LOLP) and statistical expected demand not supplied (EDNS) are adopted as the risk indices. A case study is reported to evaluate the risk posed by displacing conventional generation with wind turbines. Results on the test system show that multiples of wind turbine capacities are needed to displace conventional generation capacity to preserve the system reliability level. On the other hand, noticeable reduction is $\mathrm{CO}_{2}$ emission is achieved when wind turbines are present in the generation mix.
\end{abstract}

Keywords: Wind energy generation, generation capacity adequacy, carbon reduction

\section{Introduction}

Integration of wind energy into existing power grids remains far from easy due to its intermittency and non-dispatchability nature [1], which can significantly raise the risk of not fulfilling utilities demand obligations. The proper modelling of wind speed requires a sophisticated prospecting process. While there are several avenues to characterize the variability of a wind plant output such as the autoregressive and moving average (ARMA) model [2], the complexity of these models preclude its application in practice. To avoid such complexity, a common wind speed model, that can generate wind speed probability distributions for any geographic location and for multiple wind farm sites, if their annual mean wind speed and standard deviation of a particular site are known, is used [3].

Generating capacity adequacy indicates the ability of the system to meet the load and energy requirements. It involves combining the capacity and availability to estimate the available generation in the system. The capacity probability distribution is usually referred to as capacity outage distribution table (COPT) [4]. The COPT could be convolved with an appropriate system load representation to obtain risk measures for the generation shortfall. On the other hand, Monte Carlo simulation is a statistical tool to provide numerical estimation of the stochastic features of the system response [4]. Monte Carlo simulation can be used to incorporate wind energy into generation adequacy evaluation through suitable wind generation models.

This paper attempts to identify the risk consorted with uncertainties arising in system generation due to wind energy contribution. A common wind speed model is utilized to quantify the output power of a wind turbine generator (WTG). Probabilistic assessment is utilized to evaluate the loss of load probability (LOLP) and the expected demand not supplied (EDNS) by applying Monte Carlo simulation (MCS). The risk performance of the WTG is contemplated by displacing a conventional generation unit (CGU) with the WTG. Impact of the energy from the WTG on the $\mathrm{CO}_{2}$ emission is further highlighted.

* Manuscript received July 30, 2012; revised August 30, 2012.

Corresponding author. Tel.: +60-7-55-35335; fax: +60-7-55-36267; E-mail address: m.shaaban@fke.utm.my. 


\section{Wind Generation Modeling}

\subsection{Wind speed estimation}

Wind speed is inherently a random variable that follows a statistical distribution. Wind speed variability modelling over a lengthy period of time might not be attainable to wind farm developers due to the considerable computing power and special expertise needed. Chronological wind speed can be alternatively modelled by time series models from the actual data [2].

An Auto regressive and moving average (ARMA) time series model can be used to simulate the hourly wind speeds. Nonetheless, the complexity associated with ARMA models may hinder its application from a practical point of view [3]. A common wind speed model is used in this paper to combine different wind farm locations to generate wind speed probability distributions for any geographic location (with similar wind conditions); given that annual mean wind speed and standard deviation of the particular site are known [3].

This paper uses the normal probability distribution to model the wind speed for a particular geographical site in terms of the annual mean wind speed value $\mu$ and the standard deviation $\sigma$ [3], [5].

\subsection{Output characteristics of wind generation}

While the generated wind power varies with wind speed at the specific site, the amount of power output from a wind turbine generator (WTG) at various wind speeds can be usually characterised from wind power output curves [3], [5]. The wind power generated $(P)$ corresponding to a given wind speed $(V)$ can be obtained as [3], [5]:

$$
P=\left\{\begin{array}{cc}
0 & 0 \leq V<V_{c i} \\
P_{R}\left(A+B \times V+C \times V^{2}\right) & V_{c i} \leq V<V_{R} \\
P_{R} & V_{R} \leq V \leq V_{c o} \\
0 & V \geq V_{c o}
\end{array}\right.
$$

where

$$
\begin{aligned}
& A=\frac{1}{\left(V_{c i}-V_{R}\right)^{2}}\left[V_{c i}\left(V_{c i}+V_{R}\right)-4\left(V_{c i} V_{R}\right)\left(\frac{V_{c i}+V_{R}}{2 V_{R}}\right)^{3}\right] \\
& B=\frac{1}{\left(V_{c i}-V_{R}\right)^{2}}\left[4\left(V_{c i}+V_{R}\right)\left(\frac{V_{c i}+V_{R}}{2 V_{R}}\right)^{3}-\left(3 V_{c i}+V_{R}\right)\right] \\
& C=\frac{1}{\left(V_{c i}-V_{R}\right)^{2}}\left[2-4\left(\frac{V_{c i}+V_{R}}{2 V_{R}}\right)^{3}\right]
\end{aligned}
$$

and $V_{c i}$ is the cut-in speed $(\mathrm{km} / \mathrm{hr}), V_{R}$ is the rated speed $(\mathrm{km} / \mathrm{hr})$, whereas $V_{c o}$ is the cut-out speed $(\mathrm{km} / \mathrm{hr})$. $P_{R}$ is the rated power (MW) that is generated when the wind speed lies between the rated speed $V_{R}$ and the cut-out speed $V_{c o}$. The given wind speed $V$ can be converted to a set of wind power outputs $P$ using (1). Wind power distribution can then be created from these data. Capacity outage probability tables (COPTs) can thus be constructed including those of the WTGs.

\subsection{Multistate WTG model}

Since the output characteristics of a WTG are quite different from those of conventional generation, 
which can be adequately represented by a simple two-state model (available or unavailable), a multistate approach is used to model partial capacity states corresponding to various levels of energy output from the WTG. The WTG, in this case, is considered as a generating unit with many derated states [6].

Based on normal probability distribution, the common wind speed model is combined with the WTG output power curve (1) to create the multistate WTG model using Monte Carlo simulation as follows:

- Generate a random number for the wind speed in the range $\mu \pm 5 \sigma$ [5], [6]. This distribution ensures including extreme wind values despite their low probability of occurrence;

- Repeat the previous step until the wind speed profile is generated for the number of simulations;

- The simulated wind speed profile is plugged into the nonlinear wind output (2). Negative wind speed values have no physical significance and are converted to zero;

- Assign output states of the WTG unit as fractions of the rated power. In this paper, five states representing $0 \%, 25 \%, 50 \%, 75 \%$ and $100 \%$ of the rated power are considered. A five-state model can reasonably represent a WTG in capacity adequacy assessment [6];

- The total number of times, where wind speed results in a power output falling within one of the output states, is determined;

- Divide the total number of occurrences of each output state by the total number of simulations to estimate the cumulative probability of each state. Eventually, each state would have two parameters; the power output $P$ and its corresponding probability.

Wind farms are typically composed of many WTG units that share the same geographic location and wind speed. Since wind farms usually have identical WTGs, the multistate wind generation model will be similar to the one of a single WTG unit but with a different output power (multiples of a single unit's output).

\section{Risk indices}

The difference between the total system capacity available and the load determines the risk level in the grid system. When the load is greater than the capacity, the system is at risk. The Loss of Load Probability (LOLP) is computed to correlate the effect of generation uncertainties due to intermittent wind energy, in part, with available load. The LOLP and the expected demand not supplied (EDNS) are the load-interruption risk indices adopted in this paper [2], [7].

\subsection{Loss of load probability (LOLP)}

The loss of load probability (LOLP) is defined as the overall probability that the load demand will not be met because of load exceeding the available generating capacity under the assumption that peak load of each day lasts all day and is given by:

$$
L O L P=\sum_{i=1}^{n} P_{i}\left(C_{i}-L_{i}\right)<0 \%
$$

where

$C_{i} \quad=$ available capacity on day $i$

$L_{i} \quad=$ peak load on day $i$

$P_{i}\left(C_{i}-L_{i}\right) \quad=$ probability of loss of loads on day $i$. This value is obtained directly from the COPT.

To estimate the LOLP, sample pairs of the random variables $G$ and $L$ need to be generated. A value of the load variable $L$ is to be selected from the probability distribution of the daily peak load. First, a random number between 1 to 364 days is selected. Then the load value, in MW, corresponding to the selected random day is determined. Second, the quantity of the available generation variable $G$ is to be selected through the capacity outage probability table (COPT). The LOLP can then be computed, using the MCS, based on the occurrences that bring the demand to be greater than the generation over the total number of simulations [7]. 


\subsection{Expected demand not supplied (EDNS)}

Following the computation of the LOLP, expected demand not supplied (EDNS) can be calculated as the product of the state probability and the amount of load shortage in the system [8], [7]. An expression of the statistical EDNS is given as:

$$
E D N S=\sum_{B} P_{r}\{X=x\} \cdot\left(M W_{d}-M W_{s}\right)
$$

where

$B$ set of all unacceptable states: states where demands are not fully satisfied (e.g, exceeding the generation)

$d, s$ [demand, supply]

The LOLP and the statistical EDNS are to be calculated as measures of system risk incurred due to the incorporation of WTGs into existing conventional generating units.

\section{Numerical results}

The proposed MCS risk-based method is applied to the Roy Billinton Test System (RBTS) as shown in Fig. 1. The RBTS consists of 11 conventional generating units with a total installed capacity of 240 MW [9]. The daily load profile of the test system for 364 days (52 weeks $\times 7$ days per week) is as given in [7]. The annual peak load for the RBTS is $185 \mathrm{MW}$. It is assumed that the system originally has an adequate reliability criterion related to the balancing of demand and generation.

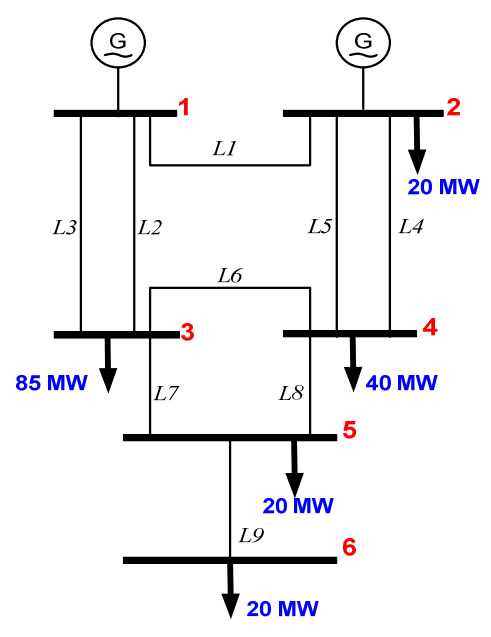

Fig. 1. The RBTS one-line diagram

Using the normal distribution of wind speed, the annual mean wind speed $\mu$ and standard deviation $\sigma$ are 19.53 and $10.06 \mathrm{~km} / \mathrm{h}$ respectively [3]. The cumulative wind probability distribution and the output levels corresponding to the five-state WTG is constructed, using MCS, as shown in Table 1. Two test cases are studied, and the risk indices are estimated by the MCS in this sequel. The number of Monte Carlo simulations is 10,000 in the proposed method.

Table 1. 5-state WTG model

\begin{tabular}{lc}
\hline Percentage (\%) & Individual probability \\
\hline 0 & 0.33127 \\
25 & 0.05815 \\
50 & 0.03845 \\
75 & 0.03822 \\
100 & 0.53391 \\
\hline
\end{tabular}




\subsection{Displacement of conventional generation}

In this case, the effect of displacing conventional generating units with sufficient identical 2 MW WTG units is investigated. A 10-MW conventional generating unit (CGU) was removed from the RBTS and displaced by five-WTG units. In this study, the base case LOLP and EDNS for the RBTS were 0.0004 per unit and 0.00065 MW respectively. Fig. 2 (a) and (b) show the LOLP and statistical EDNS against total WTG generating capacity.

An important characteristic of a generation resource is its capacity credit. Capacity credit for WTGs can be defined as the amount of WTGs output, in MW, required to replace a given amount of conventional generation output in MW, while maintaining existing levels of reliability [10].

It is clear from the graphs that 10 MW of WTGs are not matching its counterpart from the CGU as far as the reliability indices are concerned. However, a capacity credit of 28 MW or 30 MW of WTGs are the equivalent of the $10 \mathrm{MW}$ CGU since it maintains the same reliability level. In other words, almost $3 \mathrm{MW}$ of WTGs are equivalent to 1 MW of conventional capacity. Furthermore, the higher capacity credit of WTGs as compared with conventional generation are in line with the need to purchase additional transmission access rights (over nominal MW values) to facilitate integrating wind energy into existing power grids [11].

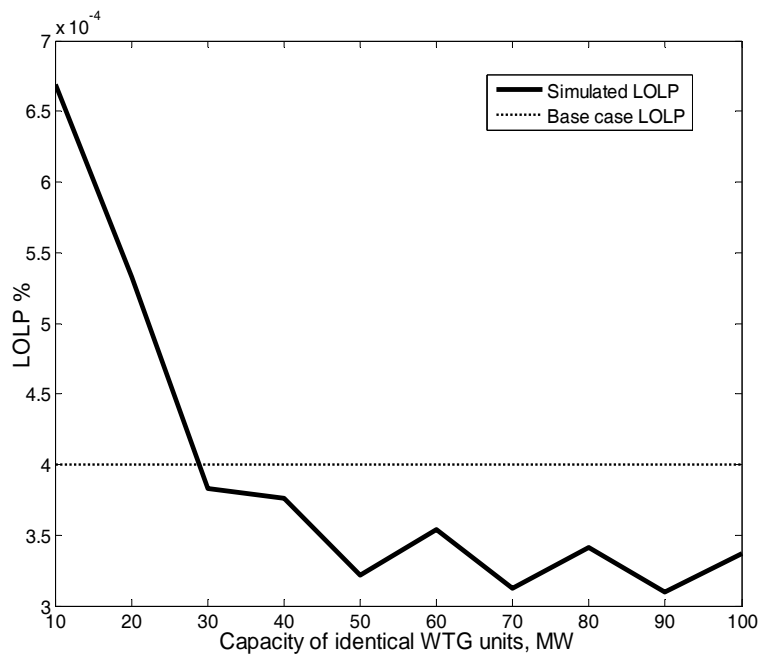

(a)

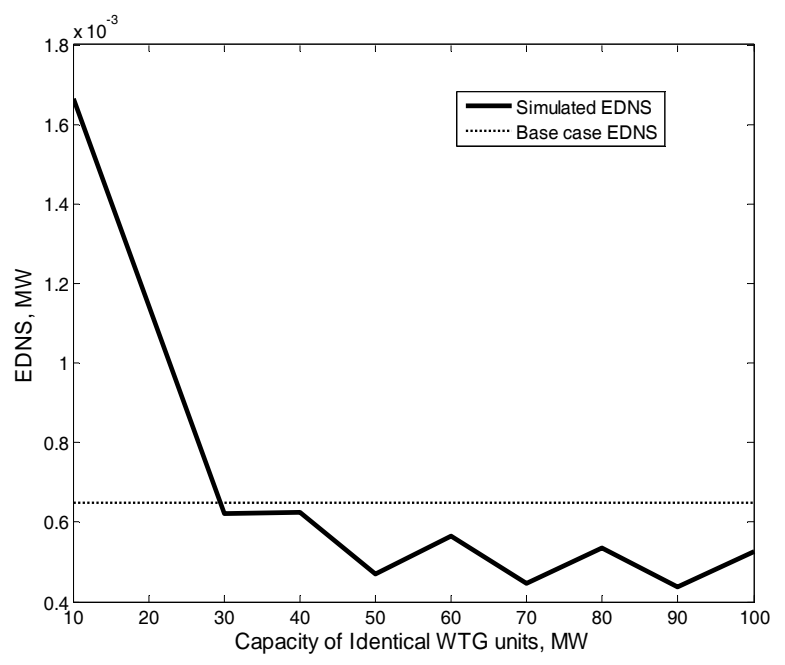

(b)

Fig. 2. (a) The annual LOLP versus total capacity of identical WTG units;

(b) Statistical EDNS (MW) against total capacity of identical WTGs

\subsection{Increase in wind penetration level}

In this case, $70 \mathrm{MW}$ of conventional generation (close to 30\% of total generating capacity) are displaced with 35 WTG units (each of 2 MW). The corresponding LOLP and EDNS have climbed up to 0.03159 per unit and $4.65879 \mathrm{MW}$ respectively. This is directly attributed to the intermittent characteristics of wind speed. The results also indicate the worsening reliability associated with deeper wind penetration in the system. The effect of higher wind penetration on carbon emission is reported. Using the formulas listed below [12], an amount of 124,777.86 tons of carbon can be abated from being released into the atmosphere by using wind energy. This is achieved by displacing $60 \mathrm{MW}$ of thermal coal units and one $10 \mathrm{MW}$ of a gas unit.

(Coal-fired) carbon emission $/ \mathrm{kWh}=0.238053 \mathrm{~kg} \mathrm{C} / \mathrm{kWh}$

(LNG) carbon emission $/ \mathrm{kWh}=0.101268 \mathrm{~kg} \mathrm{C} / \mathrm{kWh}$ 
The above results point out to the substantial environmental benefits rendered by integrating wind energy into the electricity grid.

\section{Conclusions}

This paper has investigated the risk impact of the integration of a variable energy source such as wind power on the power system dispatch. The indices used for the risk evaluation were the loss of load probability (LOLP) and the statistical expected demand not supplied (EDNS). A common wind speed model is employed and the wind generation multistate model is convolved with conventional generation respective capacities to produce the capacity outage probability table (COPT). Monte Carlo simulation (MCS) is exploited in COPT formation and reliability indices estimation. Test results on the Roy Billinton Test System (RBTS) have showed that the capacity credit of wind was about $35 \%$. On the other hand, the environmental benefit of reducing atmospheric $\mathrm{CO}_{2}$ is conspicuous. Wind power as a renewable energy source can decarbonise electric power generation and assist in reducing global warming.

\section{Acknowledgements}

This work was supported by the Malaysian Ministry of Higher Education (MOHE) and the Research Management Centre (RMC), Universiti Teknologi Malaysia, Vote No. 06J45.

\section{References}

[1] Richardson RD, McNerney GM. Wind energy systems. Proc. IEEE 1993; 81(3):378-389.

[2] Billinton R, Guang B. Generating capacity adequacy associated with wind energy. IEEE Trans. Energy Convers. 2004; 19(3):641-646.

[3] Karki R, Hu P, Billinton R. A simplified wind power generation model for reliability evaluation. IEEE Trans. Energy Convers. 2006; 21(2):533-540.

[4] Billinton R, Allan RN. Reliability Evaluation of Power Systems. 2nd ed. New York: Plenum Press; 1996.

[5] Kyeonghee C, Jeongje P, Taegon O, Jaeseok C, El-Keib AA, Shahidehpour M. Probabilistic reliability criterion for expansion planning of grids including wind turbine generators. In: Proc. of IEEE Power and Energy Society General Meeting, 2011:1-6.

[6] Billinton R, Yi G. Multistate wind energy conversion system models for adequacy assessment of generating systems incorporating wind energy. IEEE Trans. Energy Convers. 2008; 23(1):163-170.

[7] Usman MD, Shaaban M. Risk evaluation of uncertainties in generation scheduling using Monte Carlo simulation. In: Proc. of IEEE Power Engineering and Optimization Conference (PEOCO), 2012:422-426.

[8] Sharaf TAM, Berg GJ. Loadability in composite generation/transmission power-system reliability evaluation. IEEE Trans. Rel., 1993; 42(3):393-400.

[9] Billinton R, Kumar S, Chowdhury N, Chu K, Goel L, Khan E, Kos P, Nourbakhsh G, Oteng-Adjei J. A reliability test system for educational purposes-basic results. IEEE Trans. Power Syst., 1990; 5(1):319-325.

[10] Castro RMG, Ferreira AFM. A comparison between chronological and probabilistic methods to estimate wind power capacity credit. IEEE Trans. Power Syst., 2001; 16(4):904-909.

[11] Shaaban M, Bell K. Assessment of tradable short-term transmission access rights to integrate renewable generation. In: Proc. of Universities Power Engineering Conference (UPEC), 2009:1-5.

[12] Jeong SJ, Kim KS, Park JW, Lim DS, Lee SM. Economic comparison between coal-fired and liquefied natural gas combined cycle power plants considering carbon tax: Korean case. Energy 2008; 33(8):1320-1330. 